SLAC-PUB-1169

PERMANENT MULTIPOLE MAGNETIC FIELDS STORED IN SUPERCONDUCTORS*

Edward L. Garwin, Mario Rabinowitz, and David J. Frankel Stanford Linear Accelerator Center Stanford University, Stanford, California 94305

\begin{abstract}
Hollow superconducting cylinders of $\mathrm{Pb}$ and $\mathrm{Nb}$ were used to permanently store dipole and sextupole magnetic fields with high fidelity to the original fields. The fields were trapped transversely to the cylinder axes. This experimental demonstration indicates that it should be possible to store any multipole magnetic field configuration by this method. Important benefits should accrue to technologies dealing with the focussing of charged particle beams, such as accelerators, electron microscopes, etc.
\end{abstract}

(Submitted for publication.)

= Work supported by the U. S. Atomic Energy Commission. 
It has long been known that a multiply-connected superconductor can trap a magnetic field-e.g., a hollow superconducting cylinder can trap a magnetic field parallel to its axis. Previous research ${ }^{1-3}$ was primarily directed at the trapping of solenoidal magnetic fields parallel to the axis of a cylinder. To our knowledge, no one before us has stored, or suggested trapping, a multipole magnetic field transversely to the axis of a superconducting hollow cylinder. In this direction, the superconductor presents a simply-connected surface. We have successfully stored dipole and sextupole magnetic fields transversely to the axes of hollow superconducting cylinders of $\mathrm{Nb}$, as well as dipole fields transverse to both hollow and solid $\mathrm{Pb}$ cylinders, with high fidelity to the original fields. This indicates that it should be possible to store any multipole magnetic field configuration by this method. Our experiments were conducted at $4.2^{\circ} \mathrm{K}$.

We have stored fields transversely to the cylinder axis by either of the following two procedures:

\section{Procedure I:}

1. External multipole field is on.

2. Cool hollow superconductor below its transition temperature in external field.

3. Turn off external field and field remains trapped in superconductor. Procedure II:

1. Superconductor is below its transition temperature.

2. Turn on external field and drive the magnetic field into the superconductor by exceeding the critical field.

3. Turn off external field and field remains stored.

We have followed both procedures in storing dipole fields. Exceeding $\mathrm{H}_{\mathrm{c} 2}$ for $\mathrm{Nb}$ or $\mathrm{H}_{\mathrm{C}}$ for $\mathrm{Pb}$ in procedure II provided a quick way to determine the largest 
magnitude magnetic field that we could store in a given specimen. Due to limitations in the highest field that could be produced by our sextupole electromagnet, we could follow only procedure I in storing a sextupole field in a hollow $\mathrm{Nb}$ cylinder.

The $\mathrm{Nb}$ cylinders used in the experiments were $4.4 \mathrm{~cm} \mathrm{I.D.} \times 4.9 \mathrm{~cm}$ O.D. $\times 13.5 \mathrm{~cm}$ long. The field was measured by means of a number of small Hall probes, $0.25 \times 0.32 \times 0.05 \mathrm{~cm}$. In the case of the sextupole field, within the measurement error of $\pm 5 \%$, there was no detectable change in the magnitude and spatial distribution of the stored field with respect to the original field. Figure 1 shows the output voltage from one of these probes as it was rotated around the inside periphery of the $\mathrm{Nb}$ cylinder. Various magnitudes of sextupole field were stored, the large st having a magnitude of 170 Oe at a radius of $2.0 \mathrm{~cm}$ just inside the Nb cylinder. The radial dependence of the sextupole field is given approximately by $\mathrm{H}=43 \mathrm{r}^{2} \mathrm{Oe} / \mathrm{cm}^{2}$. Dipole fields were also stored in a direction perpendicular to the cylinder axis. The highest trapped dipole field was over 2600 Oe in a $\mathrm{Nb}$ cylinder. Although this maximum field reproduced the original field very well, it was metastable, and decayed with large flux jumps. Fields of approximately 2000 Oe were stably stored. Larger dipole fields ( $>2500$ Oe) were stably stored in the direction parallel to the cylinder axis. The difference may be attributable in large measure to the difference in demagnetization factor for the two directions. If this is indeed the case, then it may be possible to store larger dipole fields with cylinders of elliptical cross section, with the major axis parallel to the field. The field inside the $\mathrm{Nb}$ and $\mathrm{Pb}$ cylinders was quite uniform for the dipole case.

In the case of $\mathrm{Pb}$, both hollow and solid cylinders were used. The stored fields were stable, with a maximum field perpendicular to the cylinder axis of 290 oe, and a maximum field parallel to the axis of $500 \mathrm{Oe}$. 
The dipole field trapped transversely to the cylinder axis appears to be similar for the hollow and solid cylinders as judged by the external fields of both.

The $\mathrm{Nb}$ and $\mathrm{Pb}$ cylinders were made from high-purity stock material. We plan to further characterize our specimens by contactless induced-current resistivity measurements, as described in ref. 4. This method is ideal for the geometry of our samples, and can yield data on the Ginzburg-Iandau $\kappa$, electron mean free path, and critical fields. With stronger electromagnets, we will investigate the upper limits of a number of stably stored multipole fields for a variety of materials.

A possible explanation for the reason that a field is trapped even though the superconducting cylinder presents a simply-connected surface in a direction transverse to its axis is that the field makes the cylinder multiply-connected by creating normal regions of high flux density on a microscopic scale. The process by which this may occur, even though the field is less than $\mathrm{H}_{\mathrm{c}}$ (type $\mathrm{I}$ ) or $\mathrm{H}_{\mathrm{cI}}$ (type II) is described in ref. 5. On a macroscopic scale, the average flux density is the same as the original field because the total flux is conserved.

With the use of this method of storing multipole fields, important benefits should accrue to technologies dealing with the focussing of charged particle beams. Application to kilometer-long beam paths at the newest high-energy accelerators is inviting. Electron microscopy, in which field stability is of paramount importance, might also benefit from such technology, after detailed investigations of field fidelity and stability are carried out. 


\section{References}

1. D. G. Schwcitzer, Phys. Letters 34A, 220 (1971).

2. D. L. Coffey, W. F. Gauster, and H. E. Rorschach, Jr., Appl. Phys. Letters $\underline{3}, 75$ (1963).

3. C. P. Bean and M. V. Doyle, J. Appl. Phys. 33, 3334 (1962).

4. E. L. Garwin and M. Rabinowitz, Appl. Phys. Letters 20, 154 (1972).

5. M. Rabinowitz, J. Appl. Phys. $\underline{42}, 88$ (1971).

\section{Figure Caption}

1. Radial component of magnetic field inside Nb cylinder versus azimuthal angle showing original and stored sextupole field. 